\title{
A NEW DRAG REDUCTION METHOD BASED ON EXHAUST EMISSION TRAILING VORTEX CONTROL
}

\author{
Hua Huang \\ Guangzhou Vocational College of Technology \& Business, Guangdong 511442, China \\ Email: huanghuagztb99@163.com
}

\begin{abstract}
In order to enhance the drag reduction effect and reduce the aerodynamic drag of a small car, taking a small car as the research object, firstly, by studying the influence of the longitudinal and transverse vortices on the aerodynamic drag, the generation of the drag reduction in the tail direction is obtained. Then, according to the transient calculation method, the influence principle of the tail vortices on the drag is analysed and the method of controlling tail vortex by controlling exhaust emission is also discussed. Finally, the influence of the location and angle of the tail pipe and the shape of the tail pipe on drag reduction is studied. A new method of drag reduction using off-gas pulsating emission is proposed, and the principle of drag reduction using off-gas pulsating emission is compared with that of steady off-gas pulsating emission. The results show that when the off-gas is discharged, the drag reduction effect is certain when the different arrangement and shape of the tail pipe are considered, compared with the case without the off-gas discharging. The drag reduction effect is the most obvious when the tail pipe is at a 12-degree angle to the horizontal plane, while the circular tail pipe arranged in a certain position can reduce the drag by $3.48 \%$, and the drag reduction effect of the off-gas pulsating discharge is more obvious than that of the steady discharge, which can reach $5.38 \%$. The new drag reduction method based on exhaust emission control tail vortex can effectively alleviate the increasingly serious energy crisis, improve the utilization efficiency of automobile fuel, and improve the driving speed of automobile, so it has significant research value and broad application prospects. A new drag reduction method for exhaust emission control is studied in depth. It is very significant to reveal the principle of the influence of exhaust emission on vehicle driving speed, which is an important step to promote the use of exhaust emission to reduce vehicle aerodynamic drag.
\end{abstract}

Keywords: Exhaust Emission; Trailing Vortex; Steady Exhaust Emission; Pulsating Exhaust Emission.

\section{Introduction}

The main source of aerodynamic drag is the separation of air flow in the tail of automobile. Scientists use simulation calculation and experiment to analyze the structure of the tail flow field of automobile, and analyze the influence of the tail flow field on the aerodynamic drag characteristics, so as to reduce the aerodynamic drag by changing the flow field in the tail of automobile. Up to now, passive and active control methods have been used to reduce the drag of wake structure [1]. Passive control can reduce the aerodynamic drag by changing the flow field structure to influence the flow field around the object with corresponding control devices.

Its advantage is that it does not need additional energy, is easy to achieve, and has simple structure.

Passive drag reduction technology has completed the transformation from theory to practice, such as local body shape optimization, aerodynamic accessories such as guide vanes, spoilers, and the use of non-smooth surfaces, porous materials and so on.
Active mode can change the flow field structure around the object by applying additional disturbance according to the actual flow state in the flow field, and then achieve the purpose of reducing aerodynamic drag. Its advantage is to change the structure of the flow field by applying different excitations to the flow field without changing the shape of the object. Active drag reduction technology, including dynamic suction and jet, has been applied in some aerospace fields, but it has not been applied in automobile design yet. It is now in the stage of theoretical research, and mostly takes car-like body as the research object [2].

At present, three methods are mainly used to study the aerodynamic characteristics of automobiles: theoretical analysis, experimental research, and numerical simulation in turn.

The advantages of theoretical analysis lie in: it uses the existing theoretical basis, and by simplifying the model, it retains the main factors affecting the medium, and builds a general mathematical equation that can reflect the motion of the medium, thus 
directly revealing the inherent rules and principles of aerodynamic characteristics of automobiles [3].

However, mathematical tools and solving methods are two important difficulties in theoretical analysis. Generally, they are based on relatively simple approximation models, but it is difficult to build more complex and practical problems.

Therefore, taking theoretical analysis as the premise and basis, a feasible explanation of the problems studied is made. Emphasis is laid on the study of the aerodynamic characteristics of automobiles by numerical simulation and experimental research.

The advantage of the experimental research is that it can directly and accurately reveal the essence of fluid motion by grasping the main factors and using the test equipment to study the problems under the complete or almost similar conditions according to the similarity principle. It can be used to verify the theoretical analysis and numerical simulation of the aerodynamic characteristics of automobiles. In addition to some problems that cannot be solved in the experiment, it is often limited by objective factors such as test methods, means, equipment etc.

The numerical simulation method, also known as Computational Fluid Dynamics (CFD), is a new discipline established on the basis of classical mechanics and numerical calculation methods in the 1960s with the continuous innovation of computer technology. It has been widely used in practical engineering [4]. It focuses on the use of highperformance computers to solve various numerical simulation problems of fluid mechanics, including the simulation of fluid flow and heat transfer and other related physical phenomena. Therefore, CFD is applied to solve engineering problems such as aerodynamic characteristics of automobiles.

From the above aspects, it can be seen that the new drag reduction method using exhaust emission to control the trailing vortex as one of the direction of drag reduction for automobiles, has a good opportunity for development in the current environment. Moreover, the new drag reduction method based on exhaust emission control trailing vortex can effectively alleviate the increasingly serious energy crisis, improve the utilization efficiency of automobile fuel, and improve the driving speed of automobile, which has significant research value and broad application prospects.

\section{Numerical Simulation Theory of Automobile Tail}

The flow field of automobile tail is a complex flow field, and the external flow field is a flow field with four typical flow characteristics: three-dimensional, viscous, separation, and unsteady. This is also the most difficult point in the application of numerical simulation in automobile aerodynamics.

The shape of an automobile is regarded as a blunt body running near the ground. Its surface is a complex three-dimensional surface, and the uneven surface at the bottom of the automobile will lead to the non-periodic change of flow parameters in the direction of vehicle motion; the ground effect will occur at the bottom of the automobile, which has a unique boundary condition, and the flow parameters will change with the height of the automobile.

Therefore, in the process of three-dimensional modeling of vehicle external flow field, these factors lead to a sharp increase in the amount of calculation. At the same time, because of the existence of viscous, the boundary layer on the body surface will be generated, which will also be accompanied by the separation of the boundary layer [5].

Viscosity is not limited to the "boundary layer" of the body surface because of the uneven shape of the bottom of the car and the interference of bumpers and handlebars on the body. Therefore, when analyzing the flow field around the car, it needs to be considered as a whole, which greatly increases the number of grids of the car shape and also increases the amount of calculation. The stability and convergence of numerical simulation will be affected by the increase of mesh complexity [6].

Separation is one of the typical flow phenomena in automotive external flow field. Separation is an inevitable and complex flow phenomenon, which leads to many methods, cannot be used in the simulation of large-scale separation eddy current.

Fluid separation transfers the vorticity near the body wall to other parts of the flow field, which forms large-scale vortices. These vortices are constantly falling off the body wall and flowing downstream, and new vortices are constantly forming. From the energy point of view, these vortices are accompanied by a large amount of energy loss, acting on the body wall, and the corresponding additional drag and lift are the main manifestations. Because of the complexity of automobile flow field, there is no comprehensive or in-depth understanding of its laws and the threedimensional flow separation of automobiles. How to further study the mechanism and structure of vehicle separation flow, extract the corresponding physical and mathematical models of vehicle separation flow, and improve the accuracy of numerical simulation, is currently facing a major difficulty in the study of vehicle dynamic numerical simulation [7].

\subsection{Control direction of fluid dynamics}

CFD is a product of the combination of computer numerical calculation and fluid mechanics. Its core idea can be summarized as follows: to replace the original continuous physical quantity fields in time 
and space domain, such as temperature field and pressure field, with a set of variables separated by a series of finite discrete points, and to establish the algebraic relationship between the field variables at these discrete points by the relationship between the discrete variables, and then the numerical method is used to solve [8].

The mass conservation equation is the first law to be followed in fluid flow. The basic idea of this law can be expressed as follows: the net mass flow out of the control body through the control surface $=$ the time-varying rate of the reduction of the body mass. According to this law, the equation of mass conservation can be obtained.

$\frac{\partial \rho}{\partial t}+\frac{\partial(\rho u)}{\partial x}+\frac{\partial(\rho v)}{\partial y}+\frac{\partial(\rho w)}{\partial z}=0$

In Formula (1): $\rho$ is density; $t$ is time; $u, v$ and $w$ are the component of velocity vector $u$ in $x$, $y$ and $z$ directions [9].

In terms of the mass conservation equation of the transient three-dimensional compressible fluid given above, taking the external flow field of automobile as the research object, the compressibility of air can be neglected and the density is constant. The above equation can be simplified as follows:

$\frac{\partial u}{\partial x}+\frac{\partial v}{\partial y}+\frac{\partial w}{\partial z}=0$

Another fundamental principle of physics is applied to the flow model, namely Newton's second law.

$$
F=m a
$$

The derived equation is called momentum equation, and the law of momentum conservation is also the basic law that fluid flow must satisfy. The law can be expressed as follows: the variable of fluid momentum in a microelement is equal to the sum of forces acting on the microelement.

According to this law, the vector relation can be decomposed into momentum conservation equations along $\mathrm{x}, \mathrm{y}$ and $\mathrm{z}$ directions [10].

$$
\begin{aligned}
& \frac{\partial(\rho u)}{\partial t}+\operatorname{div}(\rho u u)= \\
& -\frac{\partial \rho}{\partial x}+\frac{\partial \tau_{x x}}{\partial x}+\frac{\partial \tau_{x y}}{\partial y}+\frac{\partial \tau_{x z}}{\partial z}+F_{x} \\
& \frac{\partial(\rho v)}{\partial t}+\operatorname{div}(\rho v u)= \\
& -\frac{\partial \rho}{\partial x}+\frac{\partial \tau_{x y}}{\partial x}+\frac{\partial \tau_{y y}}{\partial y}+\frac{\partial \tau_{y z}}{\partial z}+F_{y} \\
& \frac{\partial(\rho w)}{\partial t}+\operatorname{div}(\rho w u)= \\
& -\frac{\partial \rho}{\partial x}+\frac{\partial \tau_{x z}}{\partial x}+\frac{\partial \tau_{y z}}{\partial y}+\frac{\partial \tau_{z z}}{\partial z}+F_{z}
\end{aligned}
$$

$\tau_{x x}, \tau_{x y}$ and $\tau_{x z}$ are the components of the viscous stress $\tau$ on the surface of the microelement produced by the molecular viscous interaction.
$F_{x}{ }^{\prime} \quad F_{y}$ and $F_{z}$ : the applied forces on the infinitesimal. If only gravity acts on them, then $F_{x}=F_{y}=F_{z}=-\rho g$.

The energy equation is the third law followed by fluids. For the fluid micro-cluster model moving with fluids, this law can be expressed as: the rate of change of energy in the fluid micro-cluster = the net heat flow into the micro-cluster + the power of volume force and surface force to work on the microcluster. In the external flow field of automobile, air can be regarded as incompressible fluid because of its low velocity. Its energy equation and momentum equation can be solved separately [11].

\subsection{Mechanism of the influence of longitudinal vortices on aerodynamic drag}

As shown in Figure 1, the trailing vortex diagram of the original model is given. There is a direct relationship between the pressure difference resistance and aerodynamic drag coefficient of the trailing vortex and the vehicle at present: the pressure difference resistance of the vehicle mainly comes from the trailing vortex.

The larger the area of the trailing vortex, the higher the strength, the greater the pressure difference resistance, the more energy consumed, and the greater the aerodynamic drag coefficient.

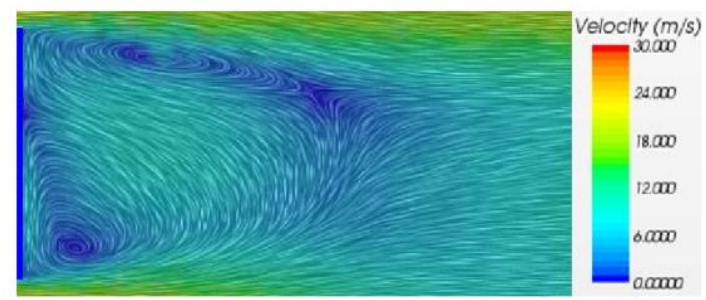

Figure 1: Prototype trailing vortex diagram

From the trailing vortex diagram of the original model, it can be seen that there are relatively strong trailing vortices at the rear of the car, which are composed of two reverse rotating vortices, one near the bottom of the car and the other at the top of the car. The phenomenon of backflow is obvious, the air flow is relatively disordered, and the area of the trailing vortex area is the largest.

\subsection{Mechanism of the influence of transverse cross-section vortices on aerodynamic drag}

The eddy field at the rear of a car not only has the eddy along the direction of the incoming flow, but also has the eddy perpendicular to the direction of the incoming flow. The eddy contains certain kinetic energy and also consumes certain energy. The resistance that the energy consumed is used to overcome is the induced resistance. From the definition of induced resistance, it can be seen that the size and intensity of the vortices determine the 
induced resistance: that is, the greater the intensity of the vortices, the greater the induced resistance.

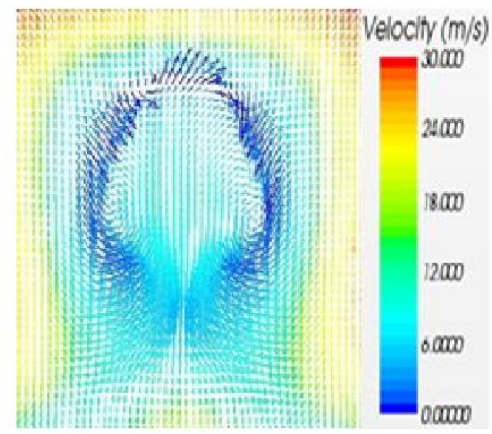

Figure 2: Velocity vector map of lateral section of vehicle tail

Figure 2 is a cross-sectional velocity vector diagram of the rear of the original model. The crosssectional distance is $0.25 \mathrm{~m}$ from the rear. It can be seen from the figure that the position of the trailing vortex of the original model is closest to the top of the car because there is no diversion plate.

A large area of low pressure area will be generated at the rear of the car. By comparing the pressure in the transverse section behind the rear of the car, the size of the vertical inflow vortices can also be measured.

As shown in Figure 3, the pressure cloud of the transverse section of the tail of the original model is taken, and the distance of the section is $0.25 \mathrm{~m}$ from the tail.

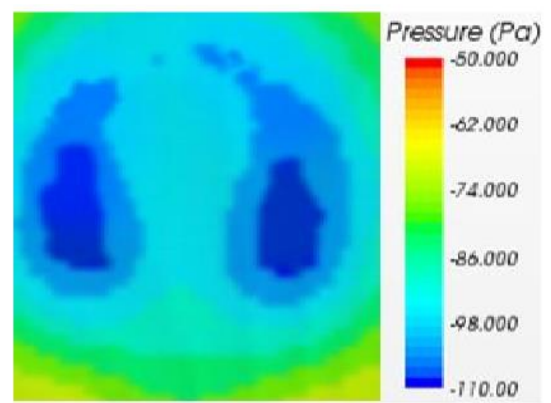

Figure 3: Pressure nephogram of rear transverse section

As can be seen from Figure 3, there are two very concentrated blue low pressure areas in the pressure nephogram at the tail of the original model.

The lowest pressure reaches $-110 \mathrm{~Pa}$, and the pressure in most areas is between $-85 \mathrm{P}$ and $-105 \mathrm{~Pa}$, which also supports the two regions of the reverse rotating eddies in Figure 1.

\subsection{Mechanism of the influence of trailing vortex on aerodynamic drag during vehicle driving}

In the course of driving, the trailing vortex state and pressure distribution at the rear of the vehicle are changing all the time, and it is the main factor affecting the aerodynamic drag. Therefore, the relationship between the structure of the shunt field and the aerodynamic drag coefficient is focused on.

Before the transient calculation, the steady-state calculation is carried out to make all residual curves reach $10^{-4}$, and the steady-state calculation results are taken as the initial values of the transient calculation. After the transient flow field is stabilized, the corresponding time is selected for analysis [12].

Figure 4 is the calculation value of aerodynamic drag coefficient after transient flow field stabilization. It can be seen that the $\mathrm{Cd}$ values of the vehicle fluctuate randomly around the average aerodynamic drag coefficient (0.31701) at different times. In order to further explore the relationship between $\mathrm{Cd}$ values and eddy currents in the near-tail region, the trailing vortex Figure 5 and pressure coefficient nephogram Figure 6 of the longitudinal symmetrical cross-section ( $\mathrm{Y}=0$ ) of the vehicle are intercepted. Additionally, four peak values of aerodynamic drag coefficients at four different times (0.851s, $1.112 \mathrm{~s}, 1.21 \mathrm{~s}, 1.435 \mathrm{~s})$ are selected for detailed analysis. The main reasons for the selection are as follows: The first is that the transient flow field is in a completely stable period after $0.5 \mathrm{~s}$.

Therefore, the selected time is reasonable. The second is that the peak value of aerodynamic drag coefficient at different times is more representative of the relationship between $\mathrm{Cd}$ value and eddy current in the near wake region.

From Figure 5 and Figure 6, it can be seen that the trailing vortices corresponding to the maximum aerodynamic drag coefficient are closest to the tail of the vehicle and have the greatest range of action, and the range of negative pressure zone is relatively large, while the trailing vortices corresponding to the minimum aerodynamic drag coefficient are far from the tail of the vehicle and have a smaller range of action, and the range of negative pressure zone is obviously reduced. Figure 5 and Figure 6 show that the reason for the high aerodynamic drag coefficient at $0.851 \mathrm{~s}$ is that two strong trailing vortices are formed at the rear of the vehicle at this time, resulting in a large negative pressure zone and a high aerodynamic drag coefficient.

At $1.112 \mathrm{~s}$, it can be found that the upper trailing vortex is obviously weakened, the lower trailing vortex is obviously pushed back and weakened, the range of negative pressure region is reduced, and the range of positive pressure region is increased, which is the main reason for the smaller aerodynamic drag coefficient.

At $1.21 \mathrm{~s}$, it is found that the range of the trailing vortex in the upper part of the vehicle increases, but the strength decreases, the trailing vortex in the lower part disappears, and the range of the negative pressure zone decreases from $0.851 \mathrm{~s}$ to $1.112 \mathrm{~s}$, which results in a higher aerodynamic drag coefficient at that time.

At 1.435s, the lower and upper trailing vortex weakens obviously, and the lower trailing vortex 
moves backward and breaks down into a very small trailing vortex.

The range of bar tropic zone is the largest, which results in the minimum aerodynamic drag coefficient at that time. Therefore, the larger the trailing vortex strength is, the larger the aerodynamic drag coefficient is.

The smaller the trailing vortex strength is, the smaller the aerodynamic drag coefficient is.

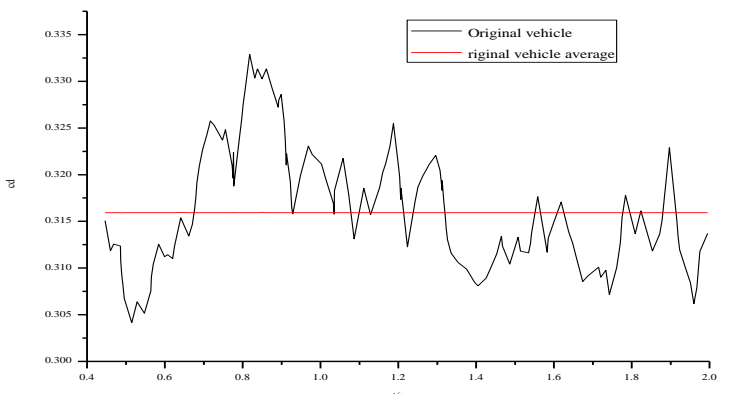

Figure 4: Aerodynamic drag coefficient of initial model at different time

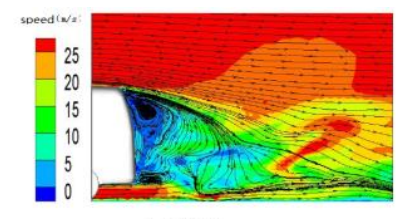

$\mathrm{t}=0.851 \mathrm{~s}$

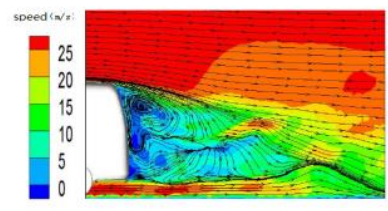

$\mathrm{t}=1.21 \mathrm{~s}$

Figure 5: Trailing vortex diagram of initial model at different time

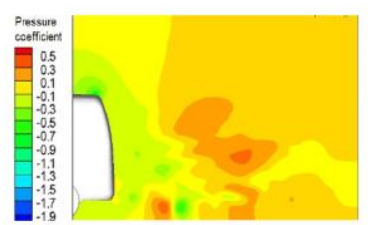

$t=0.851 \mathrm{~s}$

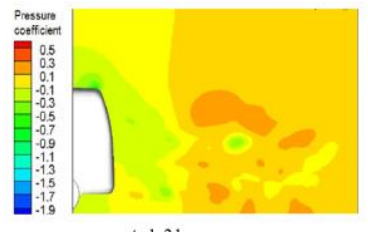

$\mathrm{t}=1.21 \mathrm{~s}$

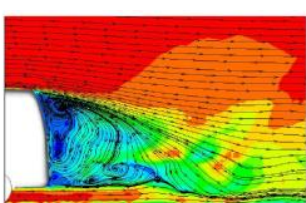

$\mathrm{t}=1.112 \mathrm{~s}$

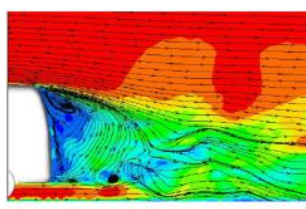

$\mathrm{t}=1.435 \mathrm{~s}$

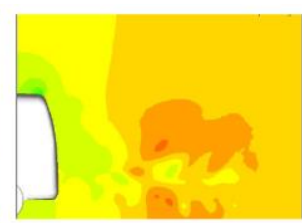

$\mathrm{t}=1.112 \mathrm{~s}$

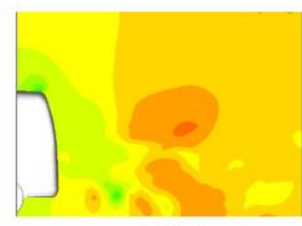

$\mathrm{t}=1.435 \mathrm{~s}$
Figure 6: Pressure coefficient nephogram of initial model at different time

\section{Effects of Exhaust Emissions and Emission Form on Aerodynamic Resistance Characteristics}

Because the exhaust emission and emission form will affect the trailing vortex of the automobile, and the trailing vortex is the main factor to produce aerodynamic drag, the reasonable exhaust emission may improve the flow field of the automobile tail, and then achieve the effect of reducing aerodynamic drag. Therefore, three-dimensional models of four typical off-gas arrangement modes are established, and CFD numerical calculation method is used to analyse the influence of exhaust emissions on aerodynamic drag characteristics [13].

\subsection{Establishment of numerical model for exhaust emission}

In UG three-dimensional modeling software, a 1:1 minibus model is established. Without affecting the overall aerodynamic characteristics of the model, proper simplification of the body of the model is made, and smaller convex devices such as wipers and rainwater tanks are omitted. The bottom of the minibus model is treated as a plane. Four typical arrangements of double exhaust circular tailpipes with a radius of $35 \mathrm{~mm}$ are established, as shown in Figure 7(a), (b), (c), (d).

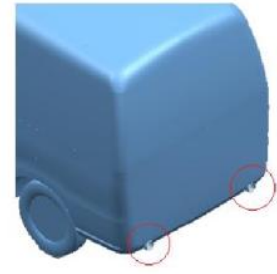

(a)

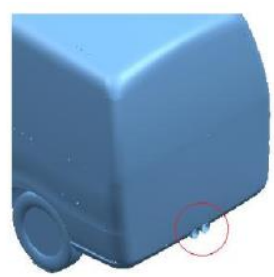

(c)

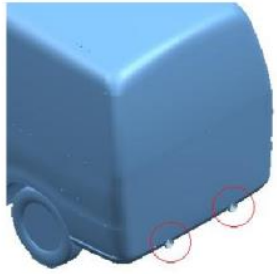

(b)

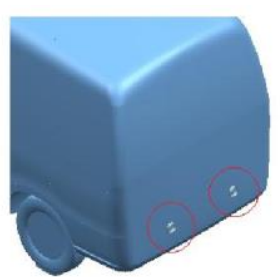

(d)
Figure 7: Vehicle model with different positions of tail pipe

\subsection{Mesh generation and boundary condition setting}

The whole mesh partition of automobile model adopts the second chapter's mesh strategy scheme 3 .

The off-gas and the atmosphere are two different attributes of the gas, so off-gas is specially treated. The off-gas gas separately establishes the space domain, while the body space domain and the off-gas gas inner space domain are connected by the interface.

The same density box is used to density the offgas in four different positions, which can not only prevent the error caused by grid inconsistency, but also ensure the accuracy of calculation.

The total number of meshes is about 5.2 million, as shown in Figure 8. 


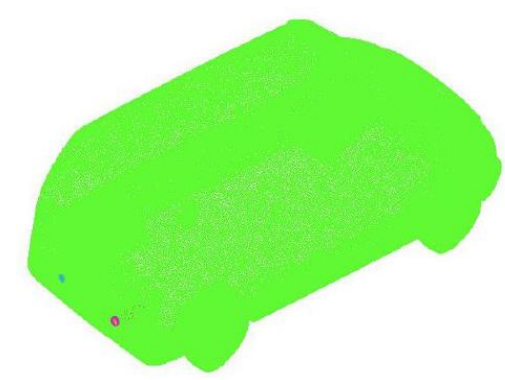

Figure 8: Model grid of vehicle and off-gas pipe

The boundary conditions are set as follows: the inlet boundary is atmospheric, the velocity is $25 \mathrm{~m} / \mathrm{s}$, the turbulence intensity is $0.5 \%$, and the density is $1.225 \mathrm{~kg} / \mathrm{m}^{3}$. The exit boundary is a standard atmospheric pressure, the body surface, the top surface and the side are non-sliding wall, the bottom is sliding wall, and the exit boundary of the off-gas pipe is simplified off-gas.

Under this condition, the flow rate of a single offgas pipe is $0.04115 \mathrm{~kg} / \mathrm{s}$, the turbulence intensity is $0.5 \%$, and the density is $0.524 \mathrm{~kg} / \mathrm{m}^{3}$.

\subsection{Analysis of calculation results}

From Table 1, it can be seen that compared with the original vehicle model, considering the influence of exhaust emissions on aerodynamic drag, the offgas pipe of minibus is arranged in the way of Figure 9 (a) (c), the aerodynamic drag is increased, and the aerodynamic drag is reduced when the off-gas pipe is arranged in the way of Figure 9 (b) (d).

From the nephogram of the turbulent kinetic energy distribution at the rear of the minibus shown in Figure 9, it can be seen that the turbulent kinetic energy at the tail of the minibus is strengthened by the arrangement of the off-gas pipe according to

Figure 9 (a). However, the turbulent kinetic energy at the rear of the minibus is weakened by the arrangement of Figure 9 (b) (d), and the turbulent kinetic energy increases, the energy dissipation increases, the aerodynamic drag increases correspondingly, and the turbulent kinetic energy decreases. The energy dissipation is also small, and the aerodynamic drag decreases accordingly.

Therefore, it can be concluded that the drag reduction method based on exhaust emission is feasible as long as the off-gas location and structural parameters are reasonably arranged.

Table 1. Effect of exhaust emission on resistance

\begin{tabular}{|c|c|c|c|c|c|}
\hline & $\begin{array}{c}\text { Original } \\
\text { vehicle } \\
\text { model }\end{array}$ & (a) & (b) & (c) & (d) \\
\hline $\mathrm{Cd}$ & 0.318 & 0.322 & 0.317 & 0.319 & 0.316 \\
\hline $\begin{array}{c}\text { Drag } \\
\text { reduction } \\
\text { rate \% }\end{array}$ & & -1.27 & 0.14 & -0.89 & 0.65 \\
\hline
\end{tabular}

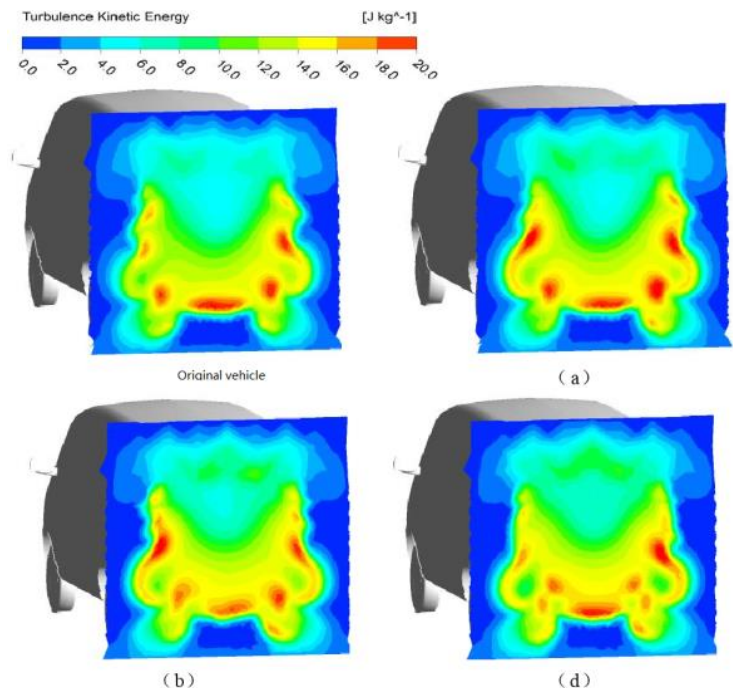

Figure 9: Nephogram of turbulent kinetic energy distribution at the tail of minibus

\subsection{Analysis of drag reduction mechanism of steady exhaust emission}

In this section, the circular tailpipe at $X=0.35 \mathrm{~m}$, $\mathrm{Y}=0.25 \mathrm{~m}$ and the ratio of exhaust emission speed to vehicle driving speed of 0.6 are selected. The mechanism of steady exhaust emission drag reduction is analyzed in detail through transient calculation, and a drag reduction method based on fluctuating exhaust emission is proposed.

Under certain conditions, it can be seen from Figure 10 that the effect of exhaust emission on reducing aerodynamic drag coefficient is obvious.

After steady exhaust emission, it is found that the aerodynamic drag coefficient at different times is significantly lower than the aerodynamic drag coefficient of the original model. Similarly, the peak values of aerodynamic drag coefficients at four different times (1.03s, 1.294s, 1.503s, 1.676s) are selected for detailed analysis, and the trailing vortex Figure 11 and pressure coefficient nephogram Figure 12 of the longitudinal symmetrical cross section $(\mathrm{Y}=0)$ of the vehicle are intercepted.

From Figures 11 and 12, it can be seen that in general, the upper trailing vortex of the car breaks into several small trailing vortices and the range of action of the vortices decreases, while the strength of the lower trailing vortices decreases obviously, and some vortices move backwards obviously.

On the whole, the range of the negative pressure zone decreases obviously, and the range of action decreases accordingly, so as to achieve the purpose of pressurization and reduce the aerodynamic drag coefficient. At 1.03s, the reason why the aerodynamic drag coefficient is larger than the other three points is that there is a large negative pressure area near the trailing vortex of rear of the vehicle, which results in a larger aerodynamic drag coefficient at that time. At 1.676s, the aerodynamic drag coefficient is smaller than the other three points 

$3.96 \%$.

because the lower and upper trailing vortices disappear basically at that time, and their action range is smaller and farther from the rear of the vehicle, and the negative pressure zone is weaker and smaller than that of the other three points, which results in the smaller aerodynamic drag coefficient at that time.

Therefore, the steady exhaust flow can destroy the structure of the trailing vortex, reduce the action range of the trailing vortex and delay the trailing vortex, thus increasing the pressure of the trailing vortex and reducing the negative pressure zone, so as to reduce the aerodynamic drag coefficient to

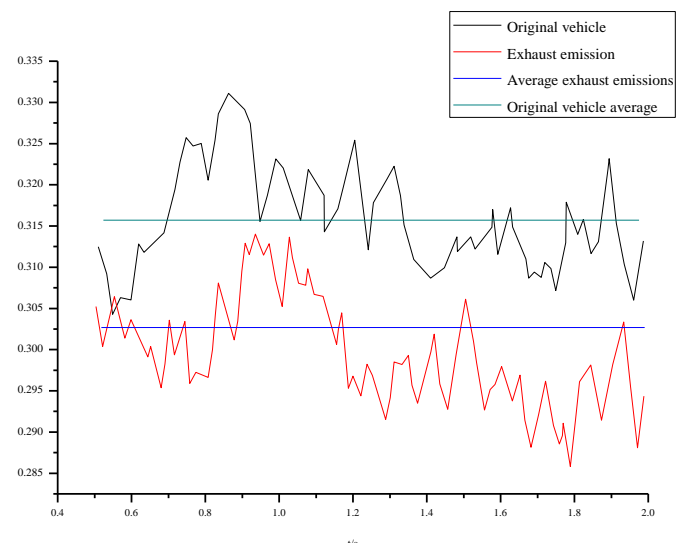

Figure 10: Aerodynamic drag coefficient at different time under steady exhaust emission

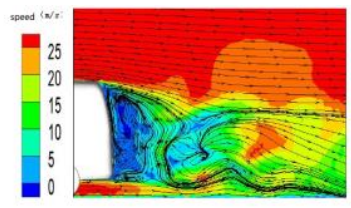

$t=1.03 \mathrm{~s}$

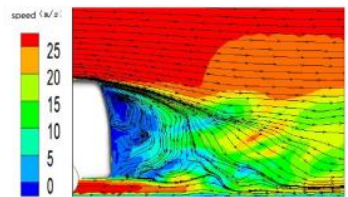

$\mathrm{t}=1.503 \mathrm{~s}$

Figure 11: Trailing vortex charts at different times under steady exhaust emission

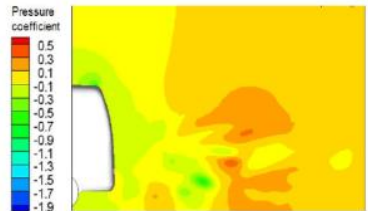

$\mathrm{t}=1.03 \mathrm{~s}$

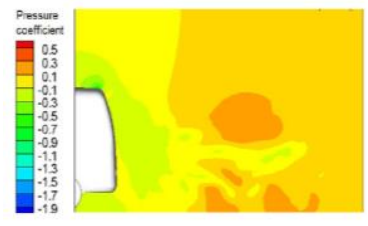

$\mathrm{t}=1.503 \mathrm{~s}$

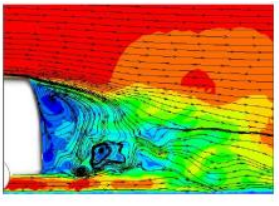

$\mathrm{t}=1.294 \mathrm{~s}$

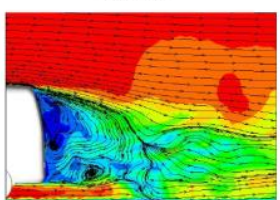

$=1.67 \mathrm{~s}$

\subsection{Analysis of drag reduction mechanism of exhaust pulsating emission}

It can be seen from Figure 13 that the drag coefficient of exhaust pulsating emission is a little higher than that of the original model at some time. It may be that the velocity of exhaust emission at this time is slightly too high, which results in a slight increase in the strength or range of trailing vortex, but the drag reduction effect is very obvious in general. The peak values of aerodynamic drag coefficients at four different times $(0.938 \mathrm{~s}, 0.977 \mathrm{~s}$, $1.018 \mathrm{~s}, 1.058 \mathrm{~s}$ ) are also selected for detailed analysis, and the trailing vortex Figure 14 and pressure coefficient nephogram Figure 15 of the longitudinal symmetrical cross section $(\mathrm{Y}=0)$ of the vehicle are intercepted.

From Figures 14 and 15, it is seen that on the whole, the action range of the upper trailing vortices of the car is obviously reduced, and the trailing vortices obviously move backward, some even disappear completely, and the strength of the lower trailing vortices increases slightly relative to the original model at some times. But on the whole, the trailing vortices of the car show a weakening trend, the range of the negative pressure zone decreases, and the range of the positive pressure zone increases, thus reducing the average aerodynamic drag coefficient of the vehicle. At 0.938s, the range of action of the upper trailing vortex is larger than that of the other three moments. Although the lower trailing vortex is far from the rear of the car, the range of negative pressure zone is larger, resulting in a larger aerodynamic drag coefficient at that time.

At 1.018, although the lower trailing vortex of the vehicle is broken into small trailing vortices at that time, the trailing vortices at that time are close to the rear of the vehicle and have a large number of intensities, and the range of negative pressure zone is relatively large, resulting in a high aerodynamic drag coefficient at that time.

At $0.977 \mathrm{~s}$, the range of action of the upper trailing vortex is obviously reduced and far from the rear of the vehicle. The strength of the lower trailing vortex is slightly increased. On the whole, the strength of the trailing vortex is weakened, the negative pressure area is greatly reduced, and the range of the positive pressure area is increased, resulting in a smaller aerodynamic drag coefficient at that time.

At 1.058s, the upper trailing vortices disappear completely, and the lower trailing vortices strength increases slightly. On the whole, the strength of the trailing vortices decreases greatly, the negative pressure area decreases greatly, and the range of the positive pressure area increases, resulting in a very small aerodynamic drag coefficient at that time.

Therefore, the exhaust pulsating emission can destroy the trailing vortex, weaken the strength of the trailing vortex, delay the trailing vortex and increase the range of barotropic zone. Under the 
same conditions, the drag reduction effect is better than that of the steady exhaust emission, reaching $5.38 \%$.

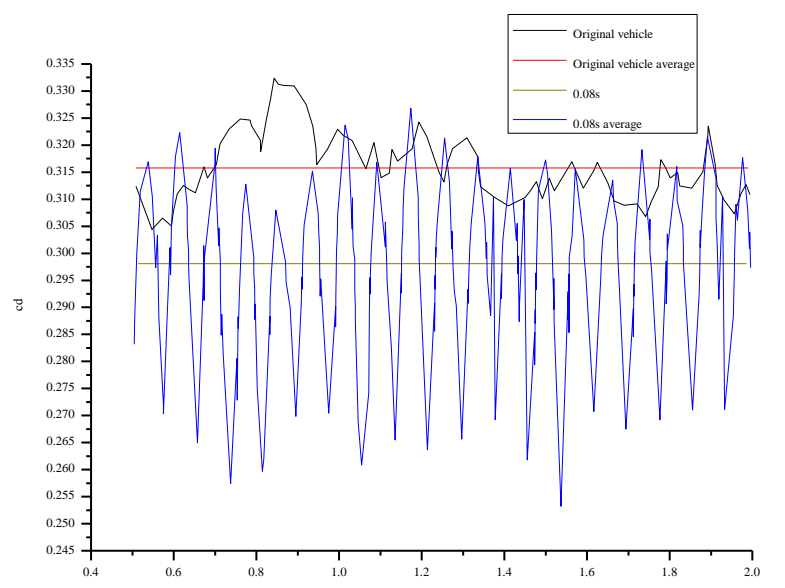

Figure 13: Aerodynamic drag coefficient at different time under steady exhaust emission

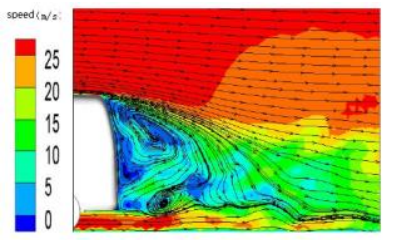

$\mathrm{t}=0.938 \mathrm{~s}$

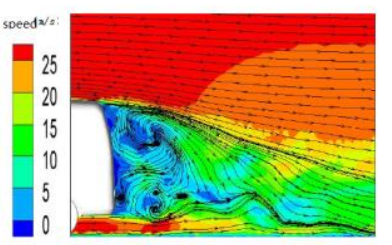

$\mathrm{t}=1.018 \mathrm{~s}$

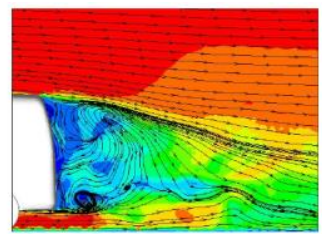

$\mathrm{t}=0.977 \mathrm{~s}$

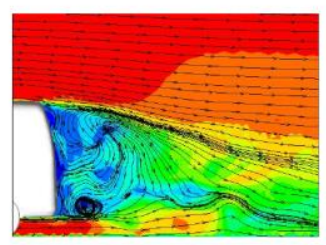

$\mathrm{t}=1.058 \mathrm{~s}$
Figure 14: Vortex charts of pulsating exhaust emissions at different times

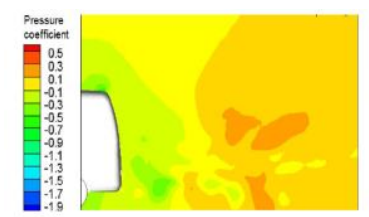

$t=0.938 \mathrm{~s}$

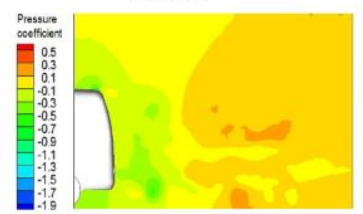

$\mathrm{t}=1.018 \mathrm{~s}$

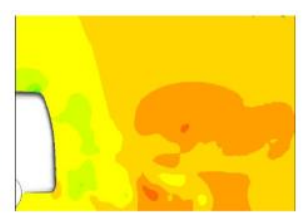

$\mathrm{t}=0.977 \mathrm{~s}$

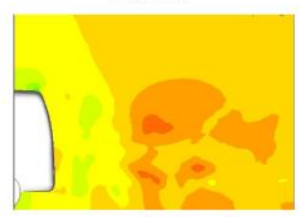

$\mathrm{t}=1.058 \mathrm{~s}$
Figure 15: Pressure coefficient nephograms at different times under pulsating exhaust emissions

\section{Analysis of Influence Factors of Tail Pipe on Drag Reduction}

Tail pipe is a necessary part of automobile. Different models have different design concepts, some are for the beauty of the car, and some are for the convenience of installation and so on [14].

The influence of exhaust gas on drag reduction from different positions, different exhaust speeds, different shapes and different angles is studied.

\subsection{Single and double tail pipe}

From Table 2, it can be seen that, compared with the original vehicle model, the tail pipe is arranged in Figure 7 (d) of the previous section.

Both single and double tail pipes can achieve the purpose of drag reduction.

However, compared with the single tail pipe, the drag reduction rate of the double tail pipe is higher with the same tail pipe flow rate and all boundary conditions, and the drag reduction rate can reach $0.64 \%$.

The main reasons are: Because the shape of the vehicle is basically symmetrical and the trailing vortex is symmetrical, the drag reduction effect of the double tail pipe is more obvious.

Table 2: Effect of single and double tail pipes on

\begin{tabular}{|c|c|c|c|}
\hline & $\begin{array}{c}\text { Original } \\
\text { vehicle }\end{array}$ & $\begin{array}{c}\text { Single tail } \\
\text { pipe }\end{array}$ & $\begin{array}{c}\text { Double tail } \\
\text { pipe }\end{array}$ \\
\hline $\mathrm{Cd}$ & 0.3171 & 0.3164 & 0.3148 \\
\hline $\begin{array}{c}\text { Drag } \\
\text { reduction } \\
\text { rate }\end{array}$ & $0.27 \%$ & $0.65 \%$ \\
\hline
\end{tabular}

\subsection{Exhaust emission velocity}

On the basis of model Figure $7(d)$, the effect of different exhaust gas velocity on drag reduction is discussed. The relationship between exhaust gas velocity and vehicle speed is expressed by $\mathrm{v} / \mathrm{u}$. When $\mathrm{u}=25 \mathrm{~m} / \mathrm{s}$, the variation of aerodynamic drag coefficient under different exhaust gas velocity on the basis of original vehicle model is obtained, as shown in Figure 16. It can be seen from the figure that the aerodynamic drag coefficient decreases in a certain range with different exhaust emission velocities. The reduction rate of aerodynamic drag coefficient varies approximately in parabolic form with the increase of exhaust emission velocities.

When $\mathrm{v} / \mathrm{u}=0.6$ or so, the reduction rate of aerodynamic drag coefficient reaches the maximum, with a drag reduction rate of $3.48 \%$. As can be seen from Figure 17, different exhaust emission velocities have great influence on the turbulent kinetic energy of the tail, which is the main reason for the difference of aerodynamic drag.

The main reason is that the trailing vortex is mainly produced by the convergence of three streams of air from the side, top and bottom of the 
car. When the exhaust emissions are considered, the trailing vortices are generated by the interaction of four streams of air.

Too low jet velocity will cause the interaction of trailing vortices to be unable to be suppressed and the trailing vortices to be destroyed; too high jet velocity will result in the formation of new trailing vortices.

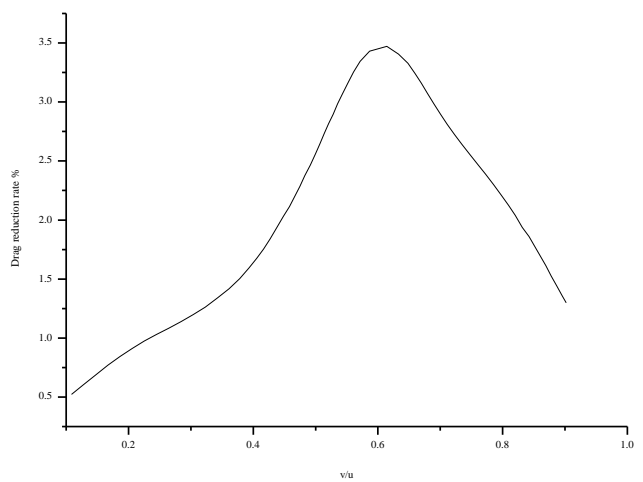

Figure 16: Reduction rate of aerodynamic resistance under different $v / u$ conditions

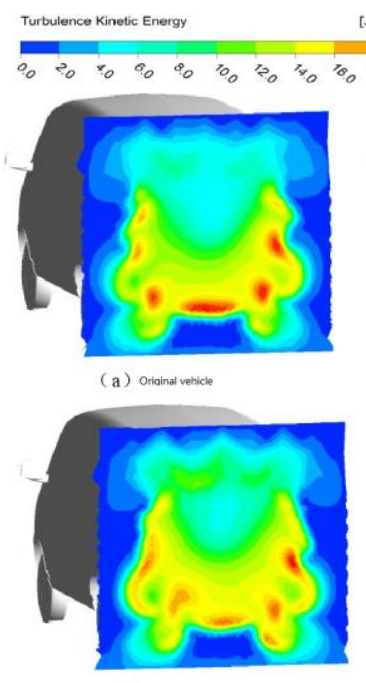

(c) $\mathrm{v} / \mathrm{u}=0.6$ $\left[\mathrm{kg} \mathrm{k}^{\wedge}-1\right]$

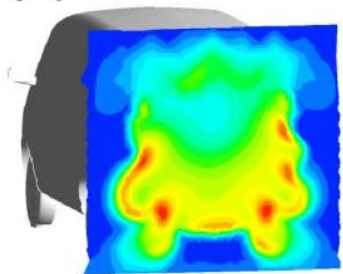

(b) $\mathrm{v} / \mathrm{u}=0.1$

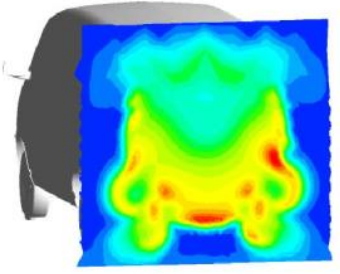

(d) $\mathrm{v} / \mathrm{u}=0.9$
Figure 17: Tail turbulent energy distribution nephogram under limit $v / u$ ratio

\subsection{Tail pipe shape}

Different tail pipe outlet shapes have great influence on aerodynamic drag, so four tail pipe shapes, circular, elliptical, square and triangle, are selected. The ratio of tail gas outlet velocity to vehicle driving velocity is set at 0.6. By comparing and analyzing the influence of different tail pipes on drag reduction, the tail pipe shape with better drag reduction effect is obtained. The four kinds of tail pipes are arranged according to Figure 7 (d). Except the shape of the tail pipes is different, all other simulation conditions are the same. The specific three-dimensional model is shown in Figure 18.
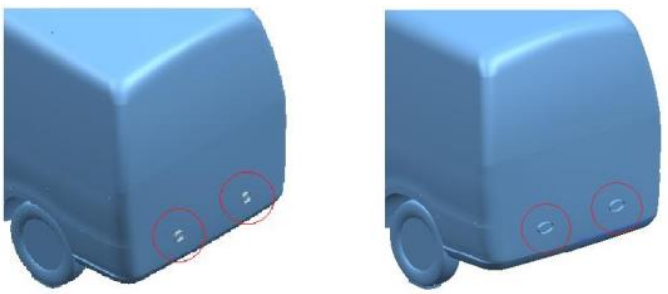

(b) circular

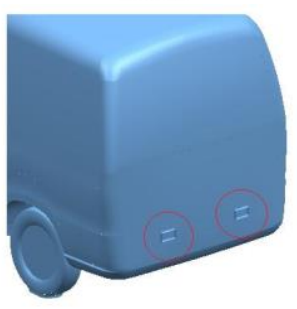

(c) Eliose

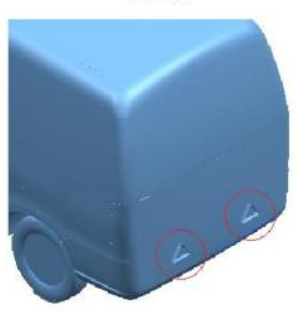

(d) souar

(e) Trianale

Figure 18: Tail pipe with different shapes

Table 3 shows the drag reduction effect of the tail pipe with different shapes. Compared with the original vehicle model, the circular tail pipe has the best drag reduction effect, with the drag reduction rate reaching $3.48 \%$, followed by the elliptical and square tail pipe. The triangular tail pipe has the worst drag reduction effect. Under the same conditions, the drag reduction rate is only $2.54 \%$.

Reason analysis: The circular and elliptical drag reduction effect is better because the shape of the exhaust jet coincides with the shape of the trailing vortex, and the offset between the two can reach the maximum, so it is more conducive to reducing aerodynamic drag.

Table. 3 Effect of tail pipe with different shape on

\begin{tabular}{|c|c|c|c|c|c|}
\hline & $\begin{array}{c}\text { Original } \\
\text { vehicle } \\
\text { model }\end{array}$ & Circular & Ellipse & Square & Triangle \\
\hline $\mathrm{cd}$ & 0.3171 & 0.3060 & 0.3074 & 0.3083 & 0.3090 \\
\hline $\begin{array}{c}\text { Drag } \\
\text { reduction } \\
\text { rate }\end{array}$ & $3.48 \%$ & $3.05 \%$ & $2.77 \%$ & $2.55 \%$ \\
\hline
\end{tabular}

Figure 19 shows the tail turbulent kinetic energy distribution nephogram of the original vehicle model and the minibus with different tail pipes. Compared with the tail turbulent kinetic energy distribution nephogram of the original vehicle model, the effect of the tail gas and the tail flow field from the four tail pipes can inhibit the formation and interaction of the trailing vortices to a certain extent, and reduce the overall turbulent kinetic energy of the tail to a certain extent. The amount dissipation is also small, thus achieving the purpose of reducing aerodynamic drag. Obviously, after installing the circular tail pipe, the tail turbulent energy of the vehicle decreases the most, so the drag reduction effect is the best, and 
after installing the triangular tail pipe, the tail turbulent energy decreases the least, so the drag reduction effect is the worst among the four tail pipe shapes.

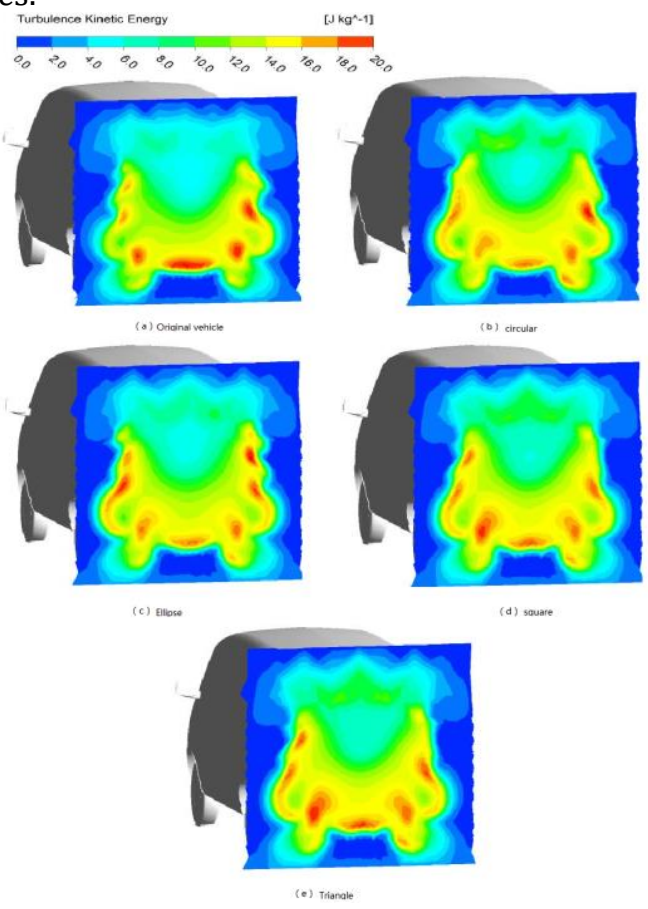

Figure 19: Nephogram of turbulent kinetic energy distribution at the tail of minibus

\subsection{Tail pipe angle}

Conventional tail pipe angle design usually adopts horizontal downward or horizontal arrangement. Because the reasonable exhaust emission can reduce drag, the angle between tail pipe and horizontal has a great influence on aerodynamic drag. Considering installation and aesthetics [15], circular double tail pipe is selected. The ratio of exhaust emission speed to vehicle driving speed is 0.6. The angle between exhaust pipe and horizontal pipe is $a$. Take one working condition every three degrees, $a$ : $-15-15$ degrees. By comparing and analysing the influence of tail pipe on drag reduction from different angles, the rule of drag reduction from tail pipe angle is found out, as shown in Figure 20.

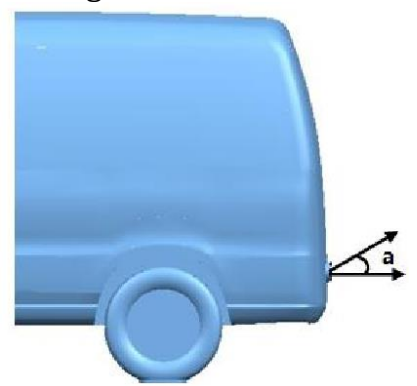

Figure 20: The angle a between the tail pipe and the horizontal plane

Figure 21 shows the reduction rate of aerodynamic drag at different angles between the tail pipe and the horizontal plane. From the figure, it can be seen that the drag reduction rate increases gradually with the increase of the angle between the tail pipe and the horizontal pipe. When the angle of the tail pipe is 12 degrees, the drag reduction rate reaches $4.4 \%$. The drag reduction rate of the tail pipe is between 12 and 15 . With the increase of the angle between the tail pipe and the horizontal pipe, the drag reduction rate decreases gradually.

Reason analysis: The interaction between the downwash of the tail vortex formed by the roof and the up wash of the tail vortex formed by the bottom of the car is stronger than that of the single interaction, and the energy dissipation increases.

When the exhaust emission can restrain the interaction between the two, the energy dissipation will be reduced, so as to achieve the purpose of drag reduction. Therefore, when the tail pipe is 12 degrees, it can restrain the downwash and up wash of the tail vortex best and reduce the interaction between them. Therefore, the tail pipe has the best drag reduction effect at this angle.

Figure 22 is a comparison of the turbulent kinetic energy distribution in the rear when the angle $a$ between the original vehicle with the tail is 12 degrees. It can be seen from the figure that when the angle of the tail pipe is 12 degrees, the turbulent kinetic energy at the bottom, top and side of the original vehicle decreases, while the range of the smaller turbulent kinetic energy in the middle increases. Therefore, the smaller the energy dissipation is, the more obvious the reduction of aerodynamic drag is.

This is mainly from this point of view, the exhaust gas can well inhibit the interaction of bottom, top and side, and reduce energy dissipation.

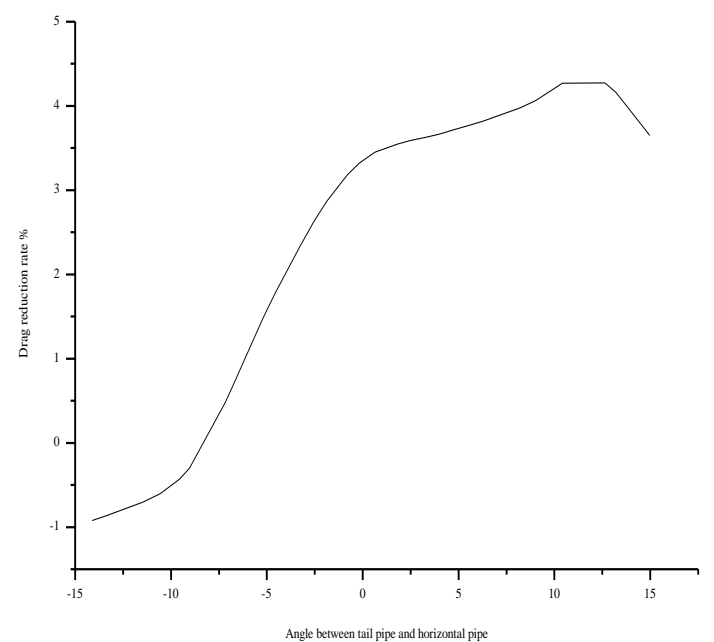

Figure 21: The relationship between drag reduction rate and different angles of tail pipe 


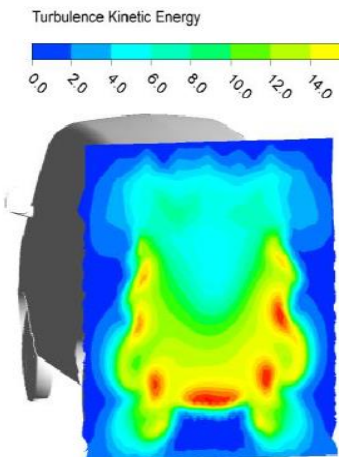

(a) Orianal vehice
[J kg^-1]

$e_{0}^{\circ}$

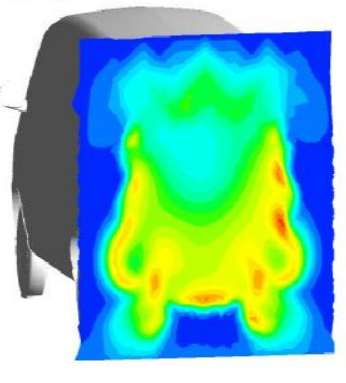

(b) a is 12 deares

Figure. 22 Comparison of turbulence energy the original vehicle and the tail is 12 degrees distribution nephogram when the angle a between

\section{Conclusion}

In the past, tail pipe is mainly designed on the basis of experience, mostly with horizontal or downward structure, and its influence on trailing vortices is not considered. A set of fast and accurate numerical simulation schemes for exhaust emission of a certain vehicle is determined firstly. Then, the influence factors of the exhaust drag reduction characteristics of the vehicle are studied, which provides a reference for the forward design of the tail pipe in the future.

A new drag reduction method for exhaust emission control is proposed. The influence of tail pipe outlet shape, angle and location on drag reduction effect is studied. The drag reduction method of exhaust pulsating emission is put forward.

The concrete conclusions are as follows: Through the transient analysis of the original model, the greater the strength of the trailing vortex and the negative pressure area, and the closer the tail is, the greater the aerodynamic drag coefficient, and vice versa. Compared with that without considering exhaust emission, the reduction of aerodynamic drag coefficient varies approximately in parabolic form with the increase of exhaust emission velocity. When $\mathrm{v} / \mathrm{u}=0.6$ or so, the reduction rate of aerodynamic drag coefficient reaches the maximum, and the drag reduction rate is $3.48 \%$. Under the same conditions, the drag reduction effect of circular and elliptical tail pipe is better than that of square and triangular tail pipe. As the angle between the tail pipe and the horizontal plane increases in turn, the drag reduction rate increases first and then decreases.

When the angle is 12 degrees, the drag reduction rate reaches $4.4 \%$. As the distance between the tail pipe and the $\mathrm{Y}$ axis increases continuously along the $\mathrm{X}$ axis, the drag reduction rate increases first and then decreases. In some locations, the drag reduction rate of the circular tail pipe can reach $3.73 \%$ at a specific location. Under these conditions, the drag reduction effect of exhaust pulsating emission is more obvious than that of steady emission. When the exhaust emission cycle is $0.08 \mathrm{~s}$, the drag reduction can reach $5.38 \%$.

With the gradual withering of natural resources, automobile aerodynamic drag reduction is becoming more and more important. The exhaust drag reduction of a certain type of vehicle is studied, and the drag reduction effect of different types of vehicle can be further considered.

The numerical model of the body surface can be further refined, especially for the bottom of the body, body accessories and so on, so that the calculation of the flow field of the automobile tail may be closer to reality.

\section{References}

[1] Fish, F. E., Legac, P., Williams, T. M., \& Wei, T. (2014). Measurement of hydrodynamic force generation by swimming dolphins using bubble dpiv. Journal of Experimental Biology, 217(2), 252-60.

[2] BRUNEAU, CharlesHenri, CREUSE, Emmanuel, GILLIERON, \& Patrick, et al. (2014). Effect of the vortex dynamics on the drag coefficient of a square back ahmed body: application to the flow control. European Journal of Mechanics, 45(2), 1-11.

[3] Mao, Blackburn, H., M., Sherwin, \& S., J. (2015). Nonlinear optimal suppression of vortex shedding from a circular cylinder. Journal of Fluid Mechanics, 775, 241-265.

[4] Yang, Y., Huang, J., Fan, G., Cai, S., \& Liu, Z. (2016). Research on non-smooth surfaces for control of the automobile trailing vortex structure. Journal of Mechanical Engineering, 52(8), 133-140.

[5] Brackston, R. D., Wynn, A., \& Morrison, J. F. (2018). Modelling and feedback control of vortex shedding for drag reduction of a turbulent bluff body wake. International Journal of Heat \& Fluid Flow, 71(C), 127-136.

[6] BRUNEAU, CharlesHenri, CREUSE, Emmanuel, GILLIERON, \& Patrick, et al. (2014). Effect of the vortex dynamics on the drag coefficient of a square back ahmed body: application to the flow control. European Journal of Mechanics, 45(2), 1-11.

[7] Manolesos, M., \& Voutsinas, S. G. (2016). Experimental study of drag-reduction devices on a flatback airfoil. Aiaa Journal, 1-15. 
[8] Auteri, F., \& Giannetti, F. (2015). Centremanifold reduction of bifurcating flows. Journal of Fluid Mechanics, 767, 109-145.

[9] Denaro, F. M. (2015). Towards a new modelfree simulation of high-reynolds local average direct numerical simulation. International Journal for Numerical Methods in Fluids, 23(2), 125-142.

[10] Haase, M., Binns, J., Thomas, G., \& Bose, N. (2016). Wave-piercing catamaran transom stern ventilation process. Ship Technology Research, 63(2), 71-80.

[11] Menezes, V. I., Takayama, K., Sun, M., Gopalan, J., \& Reddy, K. P. J. (2015). Drag reduction by controlled base flow separation. Journal of Aircraft, 43(5), 1558-1561.
[12] Modi, V. J., Shih, E., Ying, B., \& Yokomizo, T. (2015). Drag reduction of bluff bodies through momentum injection. Journal of Aircraft, 29(3), 429-436.

[13] Abdi, R., Rezazadeh, N., \& Abdi, M. (2017). Reduction of fluid forces and vortex shedding frequency of a circular cylinder using rigid splitter plates. Revue Européenne De Mécanique Numérique, 26(3), 1-20.

[14] Isaev, S., A., Baranov, P., A., Vatin, \& N., I., et al. (2014). Suppression of the karman vortex street and reduction in the frontal drag of a circular cylinder with two vortex cells. Technical Physics Letters, 40(8), 653-656.

[15] Pavia, G., Passmore, M., \& Sardu, C. (2018). Evolution of the bi-stable wake of a square-back automotive shape. Experiments in Fluids, 59(1), 20. 